\title{
Making of Mobile SunSmart: Co-designing a Just-in-Time Sun Protection Intervention for Children and Parents
}

\author{
Jimi Huh ${ }^{1} \cdot$ Kung Jin Lee ${ }^{2}$ Wendy Roldan ${ }^{3} \cdot$ Yasmine Castro $^{1} \cdot$ Saurabh Kshirsagar ${ }^{4} \cdot$ Pankhuri Rastogi $^{4} \cdot \operatorname{lan}_{\mathrm{Kim}^{1}}$. \\ Kimberly A. Miller ${ }^{1,5} \cdot$ Myles Cockburn $^{1,5}$. Jason Yip ${ }^{2}$
}

Accepted: 26 March 2021 / Published online: 13 April 2021

(c) International Society of Behavioral Medicine 2021

\begin{abstract}
Background In this study, we describe a participatory design process to develop a technology-based intervention for sun protection for children and their parents. Our methodology embraces and leverages the expert knowledge of the target users, children and their parents, about their sun protection practices to directly influence the design of our mobile just-in-time adaptive intervention (JITAI). The objectives of this paper are to describe our research procedures and summarize primary findings incorporated into developing our JITAI modules.

Methods We conducted 3 rounds of iterative co-design workshops with design expert KidsTeam UW children ( $N$ : 11-12) and subject expert children and their parents from local communities in California ( $N$ : 22-48). Iteratively, we thematically coded the qualitative data generated by participants in the co-design sessions to directly inform design specifications.

Results Three themes emerged: (1) preference for non-linear educational format with less structure,; (2) situations not conducive for prioritizing sun protection; and (3) challenges, barriers, and ambiguity relating to sun protection to protect oneself and one's family. Based on the design ideas and iterative participant feedback, three categories of modules were developed: personalized and interactive data intake module, narrative-education module with augmented reality experiment, person/ real-time tailored JITAI, and assessment modules.

Conclusions This is one of the first projects that maximally engage children and parents as co-designers to build a technology to improve sun protection with iterative and intentional design principles. Our scalable approach to design a mobile JITAI to improve sun protection will lay the foundation for future public health investigators with similar endeavors.
\end{abstract}

Keywords Co-design $\cdot$ Children $\cdot$ Parents $\cdot$ Sun protection $\cdot$ Just-in-time adaptive intervention $\cdot$ Health disparity

\section{Introduction}

Childhood and adolescence are critical periods for primary prevention for unhealthful behaviors associated with various diseases later in life [1,2]. Prevention of skin cancer

Jimi Huh

jimihuh@usc.edu

1 Department of Preventive Medicine, University of Southern California, Los Angeles, CA 90007, USA

2 Information School, University of Washington, Seattle, WA, USA

3 Human Centered Design \& Engineering, University of Washington, Seattle, WA 98105, USA

4 School of Engineering, University of Southern California, Los Angeles, CA 90089, USA

5 Department of Dermatology, University of Southern California, Los Angeles, CA 90033, USA targeting children is critical as children who incur higher ultraviolet (UV) ray exposures are at greater risk of developing skin cancer as adults [3] and sun protection practices learned at a young age tend to become lifelong habits [4]. School-based prevention interventions for children play an important role in skin cancer prevention targeted to children; however, these interventions are only moderately effective at improving sun safe behaviors and are rarely designed to incorporate approaches tailored to diverse schoolchildren [5-7].

\section{Sun Protection Interventions for Ethnic Minority Children}

While Hispanic populations tend to present with lower incidence and different histological subtypes of melanoma (i.e., acral lentiginous melanoma; ALM), the majority of their disease is histologically similar to non-Hispanic 
whites and the most common type (superficial spreading melanoma) [8]. Excessive exposure to UV radiation remains the primary modifiable risk factor for skin cancer, including ALM [9]. Ethnic minorities, including Hispanic groups, however, traditionally perceived as low risk for skin cancer, are more likely to be misinformed about their risk for sunburns and skin cancer, and tend to engage in low sun protection [10]. Even though melanoma is most common among Caucasian group, poorer outcomes have been extensively documented for ethnic minority groups $[8,11-13]$. Poor prognosis has been linked to advanced stage of disease at presentation and greater barriers to timely health care access associated with lower socioeconomic status $[8,12]$. Furthermore, recent sun protection interventions targeting children (e.g., clinic- [14, 15], technology- [16-19], or school-based [20]) often do not involve children's input in the intervention material development. However, directly involving users in the design process leads to more robust and sustainable intervention materials [21-23]. Thus, research is needed to investigate barriers for sun protection behaviors and perceived risk for skin cancer expressed by children users, which would inform the development of more effective, tailored intervention programs for diverse populations.

In this study, we describe our co-design process to develop a technology-based pilot intervention (Mobile SunSmart) for sun protection with and for children and their parents [24, 25] with diverse backgrounds, focusing on underserved groups, children and their parents with Hispanic and multiracial backgrounds [26].

\section{Importance of Involving Users: Co-designing with Children for JITAI}

Co-design is a participatory design method in which expert designers work with the target audience to solve a design problem [27]. Specifically, we adhered to the philosophy of cooperative inquiry $[25,28]$ where adults and children strive to work together as equal and equitable design partners. Two groups of children with knowledge in technology and usability ("design-domain" experts) and children with knowledge and familiarity with a given subject and contexts ("subject-domain" experts) both contributed to this process [29]. The purpose was to first understand the complexity and in-depth thoughts of the children for a starting point which we planned to later test out to a wider audience. In the cooperative inquiry method, Druin [28] emphasizes that children do not immediately become design partners from day 1 ; it takes time and effort to build the relationship. In our research, our adult design experts (KidsTeam UW) work with a small group of design-domain children for a longer period of time in which the children feel comfortable enough to share ideas and become design partners with adults. As design domain experts [29], KidsTeam UW children know the co-design techniques, understand how to co-facilitate the sessions, and are comfortable providing honest and transparent feedback. Thus, the current qualitative inquiry represents collaborative efforts among various stakeholders including child users and their parents, design experts, cancer researchers, interventionists, and mobile technology experts. The goal of this work was to develop a mobile, UV protection, prototype just-in-time adaptive intervention (JITAI) [30, 31] that is feasible and scalable, specifically designed for school-aged children and their parents with diverse backgrounds. JITAI framework maximizes available technology and allows for timely intervention delivery according to the need of the user at the moment for a given behavior $[32,33]$.

Existing primary prevention programs for sun protection among youths have been largely curriculum-based and group-delivered by health educators or teachers [3, 5, 7, 34], where timely and/or tailored dissemination is near impossible. Leveraging mobile technology such as smartphones in public health has been proliferating to aid behavioral change by promoting health behaviors (e.g., healthy eating [35]) and by curbing risky behaviors (e.g., smoking [36]). In the current study, we systematically and iteratively compiled, and pilot-tested intervention strategies suggested by the child users themselves to improve engagement and feasibility of sun protection intervention using smartphone and built-in sensors. The important risk factors for excessive sun exposure may be time-invariant (e.g., skin type, childhood sunburn history) and time-varying (e.g., UV exposure at a given time) [37-40]. These risk factors can be efficiently integrated into mobile-based interventions. Mobile-based interventions, especially those involving JITAI components, emphasize user-centeredness and tailoring based on individual cancer-related risk factors, user input, and sensor data [41-43]. The use of smartphones can allow researchers to automate the frequency of assessment of the targeted behaviors and to optimize the level and timing of tailoring, while minimizing user burden. Furthermore, smartphone platforms offer a myriad number of features (e.g., widgets, notifications) that can help improve the compliance with assessment of desired user behaviors. Similar user-centered approach has shown success in developing smartphone mobile interventions to promote sun protection designed for adult users such as mISkin (for adult vacationers in UK) [44] and Solar Cell (for US adults) [45].

Ideally, a successful JITAI would (1) target moments when the intervention deployment is deemed necessary and appropriate because those contexts are conducive of engaging in undesired behavior and (2) ensure content and timing be tailored to dynamic, real-time contexts of the user [30, 
$31,46]$. This means the technology that assesses a behavior of interest and its dynamic contexts should work seamlessly with the technology that supports the desired behaviors. It also means that the user feedback be reflected in JITAI content, medium, and timing. Thus, the ideal process for designing a JITAI to be used for children would require children users' input to minimize burden and maximize the acceptability of such technology.

Therefore, we began with the following research and design questions: (1) What type of intervention strategies and technology are perceived by our children users and their parents to be most effective and acceptable in adopting sun protection in everyday life for sustained use? (2) How can we incorporate real-time UV information to tailor to a child's personal (e.g., skin type) and real-time (e.g., being outdoors during solar peak hours) risk factors?

We engaged in co-design sessions with two groups of children with design and subject expertise [29]. Based on qualitative data obtained from the co-design sessions, we thematically coded the design ideas provided by the children and their parents [47] and incorporated them into specific design requirements for our JITAI prototypes, undergoing multiple iterations. The final selection of our strategies was guided by components and techniques proposed in mobile persuasion theories to encourage and optimize motivation, ability, and trigger, integrated with relevant gamification components for target children [48-50]. In addition, recognizing that sun protection practices among children are often initiated and managed by adult family members of the children [51], our co-design process involved children and their parents through an iterative and intentional approach. In this way, our methodology embraces and leverages the expert knowledge children and their parents have about their sun protection practices to directly influence the design of our mobile, UV-protection JITAI. We outline these design ideas and describe the corresponding prototype features in this paper.

\section{Methods}

\section{Digitizing Original In-class Curriculum Materials}

We began our co-design process with modules from SunSmart curriculum, a classroom-based curriculum that showed effectiveness in improving sun protection knowledge, attitude, and behaviors among schoolchildren [52-54]. The original SunSmart curriculum was a series of classroom-based lessons that were taught to 4 th-5th graders over 4 separate days throughout one semester (Your Skin and the Sun; What is UV Radiation; How Can We Be SunSmart?) [52-54]. The learning objectives of SunSmart were to learn about UV, learn ways to improve outdoor sun protection while enjoying the benefits of the sun, and participate in in-class activities (fruit dried in the sun; our skin and sun damage; photos of sunburn; structure of skin; damaged skin; background on UV; measuring/graphing UV index; prevention messages; does sunscreen work?; sun safe/unsafe collage). We modified the lesson plans and activities to make them suitable for the use for families and for mobile use at home. To translate the curriculum to a mobile platform, we focused on digitizing 4 of those activities because they involved direct feedback from students: solar paper, sun safe collage, lessons on UV, and prevention messages.

\section{Participants}

Design-domain Experts KidsTeam UW is an ongoing intergenerational co-design group at the University of Washington [24, 25]. Prior to the Covid-19 pandemic (2015-2020), KidsTeam UW [25] met twice a week for 90 min during the school year and they have 1-week period during summer where the children participate in all-day workshops on different design challenges. All children in the session had more than 1 year of building rapport with the adults and engaged in more than 20 co-design projects. As KidsTeam UW group works with a small group of children, the team is very intentional in recruitment on diversity considering gender, age, personality, race/ethnicity, and socioeconomic status. The children were recruited by open workshops and through interviews among children who were interested in designing technology. These children served as the designdomain youth experts [29], who engage frequently in codesign with adults (researchers, designers, graduate, and undergraduate students) and are an ethnically diverse cohort of children (50\% multi-racial, 25\% Caucasian, $8 \%$ Hispanic, $17 \%$ Asian; $N=12$ ). The co-design workshops with the same sample of KidsTeam UW children were held on three iterations in July and October in 2019 (in-person) and March 2020 (online).

Subject-domain Experts In addition to our design-domain experts, we recruited subject-domain expert children [29] from local elementary schools in the Greater Los Angeles area $(N=29)$ and their caretakers $(N=25)$. We consider these families as subject domain experts because (1) they are residents of local communities of Southern California who are knowledgeable about consistent, year-long sun exposure and their neighborhoods and (2) their demographic characteristics resemble those who will serve as our ultimate target users. To recruit subject experts, we relied on community partners to support and strengthen our connections to the local elementary schools. The investigators and research assistant, JH, YC, and KM, reached out to teachers and afterschool program directors at local elementary schools and were invited to attend parent meetings held at schools, parent 
resource centers, and afterschool programs to recruit interested parents and their families. Eligibility criteria were that participating children could read and speak in English, and there was no language restriction for participating parents. All participating children (age ranging from 8 to 12) read and spoke English, while a majority of the parents spoke Spanish. The co-design workshops with the same sample of families were held on three iterations in November 2019 (in-person) (original 22 families: 25 children and 23 parents), May 2020 (online) (subset 14 families: 15 children and 13 parents), and June 2020 (online) (subset 11 families: 12 children and 10 parents) (Table 1 ).

\section{Procedures}

The iterative co-design process $[29,55]$ involved the design experts (KidsTeam UW), subject experts (children/parents from LA communities), investigative team, and UX-programmers. All research procedures have been approved by the University of Southern California Institutional Review Board (IRB) and the University of Washington IRB.

Co-design Workshop with Design-Domain Experts We conducted initial workshops with KidsTeam UW at the University of Washington $(N=12)$ in July 2019. During these workshops, we introduced the concept of UV and the designdomain expert children participated in an outdoor activity to capture UV light onto solar paper, using various objects of their choice. Following the solar paper activity, the children mocked up ideas how concept of UV and the lessons from solar paper can be digitally translated into technologybased educational tools. Design expert children were also asked to make collages using magazine pages to represent sun protection. These activities were selected because the solar paper was a successful SunSmart activity where students were highly engaged. After participating in these activities, children provided input and suggestions on how to digitize these activities. Other discussions also included building creative technology where the children generated self-drawing curtains to protect from UV and color-changing hats corresponding to UV levels (Online Supplement Fig. 1a). In October 2019, another set of workshops were held with KidsTeam UW where they discussed the strategies to encourage continuous wear of sensors and mobile phones. They expressed challenges with complying with continuous wear of sensors especially for small devices (e.g., fear of device loss) and without tangible incentives, and suggested ways to "hack" the device. The workshop facilitators (KL, JY) also asked children how we can encourage compliance when designing ecological momentary assessment (EMA) and JIT intervention and children discussed strategies such as including image-based responses and avoiding repetitive questions. In March 2020, another iteration was executed where children discussed characters for the narratives and informed the adult co-designers that animal characters would be suitable and safe vehicles to represent diversity (e.g., skin tone), rather than human characters with different skin colors. The goal was not to generalize the 12 children's perspectives for a broad population of child-rather our epistemological interpretivist stance [56] is that the children provided designdomain expertise about multiple design features of the application which provided generative design directions for the team to present to subject-domain children.

Subject-domain Experts In November 2019, the first 2-h codesign workshops were conducted with the subject-domain expert families recruited from local LA communities. The co-design workshops were led by the investigators with codesign expertise (JY, KK, WR). The co-design sessions for the parents were led predominantly in Spanish (and interpreted in English simultaneously for English speakers). We began the workshops by emphasizing the need to engage the parents and children as co-design partners because they were the experts of their own experience. Further, we conveyed that we needed the participants' input to build a technology relevant to sun protection emphasizing that the participants themselves were the experts within their own communities and environment and how they relate to technology and sun protection. During our co-design sessions with parents, facilitators asked participants to visually sketch and
Table 1 Demographic characteristics of subjectdomain experts

\begin{tabular}{|c|c|c|c|c|c|c|}
\hline & \multicolumn{2}{|c|}{ November 2019} & \multicolumn{2}{|l|}{ May 2020} & \multicolumn{2}{|l|}{ June 2020} \\
\hline & Children & Parents & Children & Parents & Children & Parents \\
\hline$N$ & 25 & 23 & 15 & 13 & 12 & 10 \\
\hline Age, M (SD) & $9.62(1.39)$ & $37.48(8.36)$ & $10.27(1.67)$ & 39.15 (7.79) & $10.17(1.80)$ & $38(7.80)$ \\
\hline Female & $60 \%$ & $82.61 \%$ & $92.31 \%$ & $65.67 \%$ & $58.33 \%$ & $90 \%$ \\
\hline \multicolumn{7}{|l|}{ Ethnicity } \\
\hline Hispanic & $84 \%$ & $95.65 \%$ & $73.34 \%$ & $92.31 \%$ & $66.67 \%$ & $90 \%$ \\
\hline Multiracial & $16 \%$ & $4.35 \%$ & $26.66 \%$ & $7.69 \%$ & $33.33 \%$ & $10 \%$ \\
\hline Spanish preferred & -- & $73.91 \%$ & -- & $76.92 \%$ & -- & $80 \%$ \\
\hline Mother to the child & -- & $73.91 \%$ & -- & $84.62 \%$ & -- & $70 \%$ \\
\hline
\end{tabular}


narrate a day in the sun with their families. These sketches prompted collective conversations about the ways in which parents encouraged sun protection with their children. Collaboratively, parents proposed and critiqued design ideas for a mobile and wearable, UV protection, prototype that was feasible and scalable for their particular contexts (see Online Supplement Fig. 1b).

We formatted the workshop sessions so that some discussion topics would require both parents and children be present in the same room and, for other topics, parents and children participated in their respective activities in separate rooms. The rationale was that there are certain topics parents would prefer to share without their children listening, likewise children would discuss topics with their peers that they might not with their parents present. For example, in Pina et al. [57], Latino parents and children were interviewed separately to ensure each participant felt comfortable sharing sensitive information about their home experience that they normally do not share with each other. In our work, parents discussed their experiences and barriers with respect to sun protection with other parents in their preferred language (the facilitators for parents [WR and YC] were fully bilingual), another reason why we separated the parents and the children for the initial discussion as most parents chose Spanish and children chose English as their preferred language. During design time, both parents and children were presented with the initial set of design ideas provided by the design-domain expert children and were asked to discuss what/why they like/dislike about each design idea and discuss specific ways to improve them. By creating two separate groups of parents and children, we also had an opportunity to hear individual ideas in a smaller setting before going to the large group discussion. To conclude, children and parents gathered together in one room, with the investigators, and we all participated in summarizing our discussions. Bringing together the children and parents at the end was to create intergenerational opportunities for idea sharing [58]. Each family received $\$ 30$ gift card for their participation.

The co-design procedures for the two subsequent iterations (one in May 2020 and another in June 2020) were modified to be online, according to the Covid-19 restrictions for human subject research. With these necessary modifications, we preserved the integrity of cooperative inquiry principles, design materials, and presentation of design ideas by the investigative team. We used available online Zoom meeting functions to have small group discussions and met as a larger group for collective discussion to conclude. We collected artifacts by taking screenshots and video-recording the virtual sessions. More details of the format and detailed procedures of the virtual co-design and voting methods can be found elsewhere (Huh et al., under review).

\section{Data Analysis}

Following each session with the domain and subject experts, the investigators (JY, KL, YR, JH, and KM) had a debriefing session to synthesize and summarize main discussions with written notes. In addition, the artifacts produced during the workshop, including posters, photos, and audio/ video recording, were reviewed and transcribed. To analyze our rich qualitative data, we followed standard practices of qualitative research [59]. We first used inductive methods to understand the emerging themes. Four of the authors opencoded the analytic memos [60] and annotated recorded video sessions. Our team gathered to use the white board in sharing quotes and instances in the data that each researcher found interesting regarding the children's thoughts on sun protection and features in the technology. Next, we deductively compared our emerged themes to how it relates to being design design-domain expert or subject-domain expert and with technology persuasion theories. We prioritized themes that the design and subject domain groups noted. Key ideas from the memos were discussed collectively among the investigators in rounds of bi-weekly meetings and discrepancies were consolidated.

\section{Results}

Informed by insights from our co-design workshops, three overarching themes emerged. We present these underlying constructs that served as the basis for the specific design components of our JITAI intervention (see Online Supplemental Fig. 2).

The Sun Favorite activities with family while outdoor in the sun as well as adverse experiences such as sunburns were commonly mentioned. When discussing how technology can be used to teach other children about benefits of and cautions against sun exposure, a strong preference for non-linear, less-structured lessons (e.g., virtual games) was expressed. The original SunSmart curriculum aims to help children understand a variety of factors that make the concept UV ray as a "variable." Both design-domain and subject-domain expert children and parents favored being able to have control to create different scenarios. In discussing a solar paper experiment, children asked constructive and creative questions that reflected their curiosity for this topic. Based on these observations, a virtual, augmented reality experiment format where children have the control over different variables appeared be suitable for the education module.

Many children (both design and subject expert groups) were familiar with sensor technology and wanted to see automated functions (e.g., location sensing) incorporated 
to the JITAI component rather than manually providing an array of user input. At the same time, design expert children also expressed prompts and nudges by the app would turn to annoyance for users, if the app prompts with incorrect information or with great frequency.

The Situation Sharing the well-documented data that sun exposure during childhood places one at a higher risk for skin health problems later in life [61] provided an effective anchor for the co-design parents. The co-design parents discussed a variety of situations in which methods of sun protection are planned (e.g., packing a tent for a day at the beach) and others in which sun protection needs to be spontaneously devised (e.g., an unexpectedly sunny day on a biking trip or for a stroll at a swap meet market). Parents noted that they were largely responsible for their children's sun protection. All parents also expressed a desire for more detailed information about sunscreen use.

Our participants also shared many unique, cultural, and situational questions about UV lessons such as waterplay ("hiding" underwater as protection against the UV exposure; subject expert children) and home remedy for sun protection (e.g., "baby oil"; subject expert parents). Furthermore, situations involving ambiguous distinctions between temperature and UV exposure were discussed (e.g., a "hot but muggy/ cloudy" day; subject expert parents). Designing appropriate messages and intervention delivery strategies will need to consider these important cultural and situational factors. While recalling and sharing fun experiences they had as a family outdoors, both the subject expert children and parents mentioned the importance of narratives with respect to lessons and games to improve engagement for children. Subject expert children and parents expressed a strong preference for storytelling format in which SunSmart curriculum lessons are folded into meaningful narratives to communicate sun protection in a variety of contexts; this finding was consistent with existing health communication with audience with diverse background [62-64].

The Self Subject-domain expert children highlighted the need for further discussion for sun protection methods for different skin types. For instance, never having experienced sunburns was more common experience among subject expert children than having experienced sunburns. Many subject expert children and their parents discussed their perception and feelings related to their skin tone compared to some of their peers, which were often delicate topics. Some children expressed their curiosity whether the same sun protection methods applied to other children with skin types that are different from their own. More broadly, there was clear preference for cultural familiarity. Mention of familiar card games such as Loteria [65] for UV-related knowledge testing received positive feedback from both subject expert children and their parents. Other subject expert parents who were not of Hispanic background, on the other hand, suggested it could be interpreted as leaving out non-Hispanic populations. As researchers and designers, we refrain from accepting universalist frameworks which emphasize designing technological artifacts that work for everyone [66, 67]. Informed by feminist $\mathrm{HCI}$ and design frameworks [66, 67], this finding emphasizes the need to tailor and customize sun protection education and intervention messages in our mobile intervention. As such, our co-design approach with subject expert children and their parents foregrounds questions of cultural difference for sun protection.

Many children from both design and subject expert groups expressed appropriate concerns about confidentiality and privacy while using technology and sharing data. For instance, subject expert children mentioned they do not understand why they need to share their eye color on survey. However, after we explained the reason for collecting such data (i.e., melanin to determine risk factors), the subject expert children expressed they would be willing to answer those questions on the application.

With regards to "virtual" self, potential challenges and barriers to sun protection using technology were also noted by the subject expert parents. Even though most of the subject expert parents agreed that involving technology such as virtual characters (vampires and animals) was a good idea, some parents expressed concerns that relating to a virtual character may not necessarily translate to improving children's own behaviors. Some subject expert parents also mentioned that they often ensure their children are protected while they as adults might neglect or forget to protect their own skin. Most mothers in subject expert parent group mentioned that this was especially the case for their children's male caretakers. Given the particular context of our co-design sessions, however, we do not make generalizable gendered claims about the ways in which different parents navigate sun protection for themselves and their families.

\section{Corresponding Mobile SunSmart Features}

Directly informed on the co-design themes and user feedback from the iterative process, the following features have been developed for our JITAI (see Table 2 for summary).

(1) User data intake module. Information on age, gender, skin type, sunscreen, outdoor activities, and typical weekly schedules of such activities will be collected with an interactive tool. Each of these user inputs needs to be collected as we will tailor intervention messages based on the child's individual factors. Survey questions will be image-based and 
Table 2 App features and rationale corresponding to co-design discussion themes and concerns

\begin{tabular}{|c|c|c|}
\hline Themes or concerns & Reflected in the app feature as & Rationale \\
\hline Sun & $\begin{array}{l}\text { SolAR experiment } \\
\text { JITAI real-time high UV prompt }\end{array}$ & $\begin{array}{l}\text { Our subject expert children wanted UV lessons to be } \\
\text { less-structured, nonlinear experiences. SolAR makes } \\
\text { UV lessons interactive and engaging where children } \\
\text { learn that 1) UV is invisible and present when the } \\
\text { sun is present and 2) UV is variable, influenced by } \\
\text { environmental factors (e.g., time of the day, cloud } \\
\text { coverage, exposure duration), which is additionally } \\
\text { reinforced by high-UV JITAI warning prompt }\end{array}$ \\
\hline Situation & 7 Episodes folded into Marco \& Nelly narratives & $\begin{array}{l}\text { The subject expert children and their parents agreed that } \\
\text { storytelling can be a powerful health communication } \\
\text { tool that can seamlessly address different scenarios } \\
\text { and contexts. Research has shown that the storytell- } \\
\text { ing medium has been particularly effective among } \\
\text { Hispanic groups [62] }\end{array}$ \\
\hline Self & Image-based survey for personal tailoring & $\begin{array}{l}\text { Colorful, virtual images were displayed to aid children } \\
\text { while responding to survey to minimize ambiguity of } \\
\text { items (e.g., colors of skin: light brown, eye: hazel) }\end{array}$ \\
\hline Privacy and confidentiality & $\begin{array}{l}\text { Information icon for app feature functions and reasons } \\
\text { for collecting personal data }\end{array}$ & $\begin{array}{l}\text { Children were curious why they need to provide certain } \\
\text { data (e.g., eye color) or app functions (e.g., location } \\
\text { detection) that could seem invasive. Providing a fea- } \\
\text { ture with further information empowers children users } \\
\text { and minimizes potential suspicion or mistrust }\end{array}$ \\
\hline Gamification & $\begin{array}{l}\text { Badges and trophies for completing videos and AR } \\
\text { experiment }\end{array}$ & $\begin{array}{l}\text { Badges and trophies are content-based and represent } \\
\text { each activity where users can visualize their "achieve- } \\
\text { ment" in their learning process. Children can collect } \\
\text { all the badges and trophies corresponding to each } \\
\text { feature }\end{array}$ \\
\hline SunSmart Education booster & Vampire Trail & $\begin{array}{l}\text { Children wanted a light video game to increase daily } \\
\text { engagement with the app; user can help a virtual } \\
\text { SunSmart character earn points by daily check-in, } \\
\text { Vitamin D maintenance, outdoor fun-with-family }\end{array}$ \\
\hline User burden & $\begin{array}{l}\text { JITAI to be triggered by geofencing and geolocation } \\
\text { API }\end{array}$ & $\begin{array}{l}\text { We were mindful that user burden introduced by this } \\
\text { algorithm be minimized. We ensured that JITAI trig- } \\
\text { gers be deployed only during solar peak hours and only } \\
\text { after } 20 \text { min of continuous outdoor location is detected }\end{array}$ \\
\hline
\end{tabular}

child-friendly and have been approved by our subject expert children during co-design sessions. To address the confidentiality and privacy concerns shared by subject expert children and parents, each survey question will be accompanied with an "information" icon to explain as to why we ask such questions (Online Supplemental Fig. 3). This was to address privacy concerns that our subject expert children raised during our co-design sessions.

(2) Narrative-based curriculum module. Our codesign participants (both children and parents) provided highly positive feedback on sun protection lessons embedded in storytelling format that addresses extension of self and various situations, a narrative in which they can relate to and can "see themselves" as protagonists in the story. Our narrative tells a story of two siblings, Marco and Nelly, and their family and friends (Online Supplemental Fig. 4) as the main characters who navigate through different scenarios based on the various themes that surfaced from our co-design workshops. The focus is to couch educational materials into the narratives of Nelly and Marco [68-70] in seven episodes in a video format to which children are already accustomed. We introduced the storyline to our children co-designers before we began constructing scripts. With their feedback, we revised the scripts and the images iteratively. Two subcomponents (virtual experiments and curriculum booster) are folded into the main narrative.

(2a) Augmented reality (AR) virtual solar paper experiment that allows users to experiment with factors that affect UV exposure (Online Supplemental Fig. 5). Based on the positive feedback on interactive, "non-classroom like" learning, the main lessons of this feature are to teach children (1) that UV radiation is invisible and (2) UV-related factors are variable that can be virtually manipulated by the users, 
namely, UV strength, time of the day, seasonality, longitude, and latitude. Manipulating these variables encourages users to engage in experimental design and observe the changes in a digital world to then be more aware of UV-related factors in the real world. To maximize the engagement by child users, users will be able to point the smartphone camera at any background or object and the physical object solar paper is replaced by AR virtual paper whose color will change per virtual manipulation by the user. This way, the mechanisms by which color of the object changes would become more explicit. During codesign sessions, most of our participants from both design and subject expert groups expressed keen interest in playing with the AR feature to observe the outcomes of changing variables.

(2b) A virtual game ("Vampire Trail") that reinforces the importance of enjoying the benefit of the sun, while exercising sun protection. The goal of this daily game is to gain the most sun protection, fun, and vitamin $D$ points while increasing engagement with the app. Users will be encouraged to use these features as they interact with the Mobile SunSmart suite and the usage data for each feature will be logged. The usage data for each feature will be analyzed to quantify the usability and engagement levels with individual features of the app.

(3) Personal and real-time tailored, just-in-time module. The main function of this module will be to deploy the main JITAI intervention based on the algorithm that combines person-level (from user data intake) and real-time UV and geofence data. Our system sets up virtual boundaries based on users' geolocation data collected by smartphone in real time and tags areas enclosed in a given geofence as indoor or outdoor based on the type of the location (e.g., the boundaries of one's home are considered a geofence and would be tagged as indoor whereas the boundaries of a playground are considered as a geofence, tagged as outdoor). The app will trigger pre-programmed actions such as sending mobile notification when pre-defined boundaries or geofences are crossed [71, 72].

(3a) Daily prevention. Preemptive prevention messages will be sent out every morning. The messages will be tailored based on child's skin type, UV forecast for the day and outdoor activity planned for the day.

(3b) JIT intervention. JIT messages will be sent out when exposure exceeds predetermined thresholds for a given child (i.e., approximate number of minutes of UV exposure during solar peak hours, $10 \mathrm{am}-4 \mathrm{pm}$ ). Person-level and time-varying data such as skin type, real-time UV index at user's current location, out- door/indoor location determined by geofence data, and the user's sunscreen SPF value will be combined to calculate the Safe Exposure Time (SET, defined as $10 \mathrm{~min}$ before the approximate minimum time to sun damage such as suntan or sunburn at a given moment). The mobile device sends a notification to remind the users to engage in sun protective behaviors after the SET is elapsed, thus providing JIT intervention. (3c) End-of-day (EOD) assessment. Daily self-report of sun protective behavior will be collected via interactive methods, leading to earning badges. Users can earn "streak" badges with completing EOD for multiple days in a row, for instance. We believe these interactive methods will increase the EMA compliance. The importance of confidentiality and integrity of sharing one's own behavioral data will be conveyed to child users and their guardians throughout.

\section{Discussion}

In this paper, we describe our co-design process that involved children and their parents through an iterative and intentional approach to design a JITAI mobile SunSmart intervention. Existing sun protection interventions for children often overlook disease-specific risk factors and culturally unique factors. Most of these programs often do not focus on co-developing strategies to optimally intervene for sustained changes. Such efforts have been particularly scant for children and families with cultural diversities. Our methodology maximally leveraged multiple stakeholders [24, 25, 29, 57, 73], most importantly the expert knowledge of the children and their parents about their own sun protection practices to directly influence the design of our JITAI.

To meet the unique set of design needs among our target users, we engaged children with design and subject expertise and their parents who provided design ideas [24, 29]. This iterative process led us to correct and refine our initial speculations about what technology and strategies would be useful and feasible given their relevant contexts. The discussion based on ethnically and socioeconomically diverse participants' backgrounds and experiences helped us understand that narratives-based content with a video format would be well-received and most scalable for sun protection-related education. Children and parent co-designers shared concerns that every situation they find themselves in is not always ideal or convenient for prioritizing sun protection. They identified challenges, barriers, and ambiguity relating to sun protection, especially for children with different skin tones within a family. The co-designer children and parents also pointed to the lack of discussion and confusion regarding sun protection for different skin types. The iterative discussions also highlighted 
that not all technological artifacts would be equally suitable for everyone or across all contexts. Our research approach not only surfaced these unique insights from our participants that researchers have not previously identified, but also directly translated their qualitative insights into digital features for a mobile application.

Based on the design ideas and iterative feedback provided by our participants, we have developed three modules: personalized and interactive data intake module, narrative-education module, person-level and real-time tailored JITAI, and assessment module. Each module and individual features within each module have been guided by a set of engagement strategies that our participants informed us to be effective (i.e., the dos and don'ts). In future work, we plan to pilot test the beta version of the prototype JITAI with an independent sample of child users. To note, the level of enthusiasm of the local community families for providing feedback and remaining to be part of the study was remarkable.

We have noted several barriers and new information that were highlighted during the iterative co-design stages. For instance, sun collage activity that had been well-received as part of the classroom-based curriculum was not suitable for digital translation (e.g., "too classroom like"). Weaving in a storyline of Hispanic families in local settings to discuss sun protection issues was important to make the issue more directly relevant to ethnic minority children rather than to alienate them. Our JITAI design will also benefit from the children's feedback that adding a wearable will be too burdensome for the users, which led us to find an alternative to achieve the same goal by combining geofence data and real-time UV data. We further learned that relying on calendar input to assess daily outdoor activity was also not feasible due to user burden. Therefore, we learned that Mobile SunSmart JITAI needs to be much more than a direct digital translation of classroom-based, "analogue" materials.

Reflecting on our methodological approach, we encountered a barrier when discussing sun protection with the children and the family because the topic of skin cancer was not a salient one. Highlighting the skin cancer disparity-related facts sparked interest and facilitated the discussion among our participants. We encourage other researchers to engage in similar approaches in addressing health issues that may be difficult to raise or may not be immediately salient among community members. Based on our experience, we also emphasize that building rapport with each of our participant group of co-design families was critical. Some of the subject expert children and family members were willing to participate in codesign iterations with much enthusiasm even during incredibly challenging time of Covid-19 during Spring 2020 with many constraints as they felt that they were part of the team.

Our findings highlight the importance of involvement of user input from the beginning and throughout the iterative process of intervention development for sun protection. This point is crucial as children and their parents, especially those with diverse and marginalized backgrounds in particular, have been often left out in the process of mobile health tool development [35]. As we have learned during our co-design process, children provide insightful information ranging from message delivery medium to specific user experience/ interface designs as sophisticated technology and information consumers. Furthermore, not surprisingly, our participants' familiarity with technology has grown more apparent as children became increasingly accustomed to online learning modifications due to Covid-19 restrictions. In sum, input from our subject expert children and parents served a critical role as we intended to develop technology that needs to be relevant and useful for the users [24, 25, 27, 29, 73].

\section{Limitations}

There are several limitations of our study. Our research methods focused on subject expert children and parents from ethnically diverse communities in Southern California and the later phase of our co-design sessions were completed during Covid-19 pandemic. Therefore, the qualitative data from codesign workshop may not be generalizable to other samples with different backgrounds or to all other temporal contexts. Mobile technology-related limitations are also noted. User perceptions about the proposed technology may change as the actual application becomes widely available. Preference for various modules and features might differ depending on app functionality and socioeconomic covariates such as gender, ethnic background, and resources. Furthermore, the app will be developed in Android; therefore, users more familiar with iOS might exhibit different usage patterns than Android users. Lastly, children will be asked to interact with the system installed on project phones for 2 weeks. Even though a majority of elementary school-aged children would be familiar with smartphones, interacting with an app on a smartphone can be distracting and burdensome. We will work with our participants to minimize such potential issues by disabling other features on project devices.

\section{Conclusion}

Despite these limitations, Mobile SunSmart is one of the first projects that maximally engage children co-designers and parents to build a technology to improve sun protection for those users with iterative and intentional design principles. The design process was informed by children and their families, public health, health communication, and human-computer interaction (HCI) theories. We hope that our scalable approach to design a mobile JITAI to improve sun protection will lay the foundation for future public health investigators for similar endeavors. 
Supplementary Information The online version contains supplementary material available at https://doi.org/10.1007/s12529-021-09987-9.

Acknowledgements We are truly grateful to all our co-design participants who shared with us their ingenious ideas and precious time. We thank the USC Joint Educational Program and teachers from partner public schools in Los Angeles for their generous assistance. We also thank Dr. Mojgan Sami from Cal State University of Fullerton and members of the non-profit organization, Scientastic, Inc., for their brilliant creativity and execution on the audio/video production of the narratives.

Funding This study was supported by the National Institute of Environmental Health Sciences (1R21ES029570-01A1, PI: Huh). The content is solely the responsibility of the authors and does not necessarily represent the official views of the National Institutes of Health.

\section{Declarations}

Ethical Approval All procedures performed in studies involving human participants were in accordance with the ethical standards of the institutional and/or national research committee and with the 1964 Helsinki declaration and its later amendments or comparable ethical standards.

Informed Consent Informed consent was obtained from all adult individual participants included in the study and assent form was obtained from all children participants included in the study.

Conflict of Interest The authors declare no competing interests.

\section{References}

1. Milne E, et al. Improved sun protection behaviour in children after two years of the Kidskin intervention. Aust N Z J Public Health. 2000;24(5):481-7.

2. Whiteman DC, Whiteman CA, Green AC. Childhood sun exposure as a risk factor for melanoma: a systematic review of epidemiologic studies. Cancer Causes Control. 2001;12(1):69-82.

3. Saraiya M, et al. Interventions to prevent skin cancer by reducing exposure to ultraviolet radiation: a systematic review. Am J Prev Med. 2004;27(5):422-66.

4. Holman DM, Watson M. Correlates of intentional tanning among adolescents in the United States: a systematic review of the literature. J Adolesc Health. 2013;52(5):S52-9.

5. Buller DB, et al. Evaluation of the Sunny Days, Healthy Ways sun safety curriculum for children in kindergarten through fifth grade. Pediatr Dermatol. 2006;23(4):321-9.

6. Emmons KM, et al. The SunWise Policy intervention for schoolbased sun protection: a pilot study. J Sch Nurs. 2008;24(4):215-21.

7. Tripp MK, et al. Children's skin cancer prevention: a systematic review of parents' psychosocial measures. Am J Prev Med. 2013;44(3):265-73.

8. Pollitt RA, et al. The expanding melanoma burden in California Hispanics: importance of socioeconomic distribution, histologic subtype, and anatomic location. Cancer. 2011;117(1):152-61.

9. Sample A, He YY. Mechanisms and prevention of UVinduced melanoma. Photodermatol Photoimmunol Photomed. 2018;34(1):13-24.

10. Lunsford NB, et al. Skin cancer knowledge, awareness, beliefs and preventive behaviors among black and Hispanic men and women. Preventive medicine reports. 2018;12:203-9.
11. Garnett E, et al. Characteristics, rates, and trends of melanoma incidence among Hispanics in the USA. Cancer Causes Control. 2016;27(5):647-59.

12. Harvey, V.M., et al., The role of neighborhood characteristics in late stage melanoma diagnosis among hispanic men in California, Texas, and Florida, 1996-2012. J Cancer Epidem, 2017.

13. Rouhani $\mathrm{P}, \mathrm{Hu} \mathrm{S}$, Kirsner RS. Melanoma in Hispanic and black Americans. Cancer Control. 2008;15(3):248-53.

14. Crane LA, et al. Mailed intervention to promote sun protection of children: a randomized controlled trial. Am J Prev Med. 2012;43(4):399-410.

15. Glasser A, et al. The sun sense study: an intervention to improve sun protection in children. Am J Health Behav. 2010;34(4):500-10.

16. Dudley DA, et al. Rationale and study protocol to evaluate the SunSmart policy intervention: a cluster randomised controlled trial of a primary school-based health promotion program. BMC Public Health. 2015;15(1):42.

17. Hingle MD, et al. Effects of a short messaging service-based skin cancer prevention campaign in adolescents. Am J Prev Med. 2014;47(5):617-23.

18. Ho BK, et al. Effectiveness of a multicomponent sun protection program for young children: a randomized clinical trial. JAMA Pediatr. 2016;170(4):334-42.

19. Olson AL, et al. SunSafe in the middle school years: a communitywide intervention to change early-adolescent sun protection. Pediatrics. 2007;119(1):e247-56.

20. Reinau D, et al. Sun protective behaviour of primary and secondary school students in North-Western Switzerland. Swiss Med Wkly. 2012;142:w13520.

21. Banbury A, et al. Adding value to remote monitoring: co-design of a health literacy intervention for older people with chronic disease delivered by telehealth-The telehealth literacy project. Patient Educ Couns. 2020;103(3):597-606.

22. Champion KE, et al. A web-based intervention to prevent multiple chronic disease risk factors among adolescents: co-design and user testing of the Health4Life school-based program. JMIR Formative Research. 2020;4(7):e19485.

23. O'Brien $\mathrm{N}$, et al. Integrating evidence from systematic reviews, qualitative research, and expert knowledge using co-design techniques to develop a web-based intervention for people in the retirement transition. J Med Internet Res. 2016;18(8):e210.

24. Yip, J.C., et al. The evolution of engagements and social bonds during child-parent co-design. in Proceedings of the $2016 \mathrm{CHI}$ Conference on Human Factors in Computing Systems. 2016. ACM.

25. Yip, J.C., et al. Examining adult-child interactions in intergenerational participatory design. in Proceedings of the 2017 CHI Conference on Human Factors in Computing Systems. 2017. ACM.

26. Hu S, et al. Disparity in melanoma: a trend analysis of melanoma incidence and stage at diagnosis among whites, Hispanics, and blacks in Florida. Arch Dermatol. 2009;145(12):1369-74.

27. Walsh, G., et al. FACIT PD: a framework for analysis and creation of intergenerational techniques for participatory design. in Proceedings of the SIGCHI Conference on Human Factors in Computing Systems. 2013.

28. Druin A. The role of children in the design of new technology. Behaviour and information technology. 2002;21(1):1-25.

29. Yip, J., et al. Brownies or bags-of-stuff?: domain expertise in cooperative inquiry with children. in Proceedings of the 12th International Conference on Interaction Design and Children. 2013. ACM.

30. Nahum-Shani, I., E.B. Hekler, and D. Spruijt-Metz, Building health behavior models to guide the development of just-in-time adaptive interventions: A pragmatic framework. Health Psychology, 2015. 34(S): p. 1209. 
31. Nahum-Shani I, et al. Just-in-time adaptive interventions (JITAIs) in mobile health: key components and design principles for ongoing health behavior support. Ann Behav Med. 2018;52(6):446-62.

32. Goldstein SP, et al. Return of the JITAI: applying a just-intime adaptive intervention framework to the development of $\mathrm{m}$-health solutions for addictive behaviors. Int J Behav Med. 2017;24(5):673-82.

33. Intille, S.S., et al. Just-in-time technology to encourage incremental, dietary behavior change. in AMIA Annual Symposium Proceedings. 2003. American Medical Informatics Association.

34. Buller DB, et al. Effects of the Sunny Days, Healthy Ways curriculum on students in grades 6 to 8. Am J Prev Med. 2006;30(1):13-22.

35. Curtis KE, Lahiri S, Brown KE. Targeting parents for childhood weight management: development of a theory-driven and user-centered healthy eating app. JMIR Mhealth Uhealth. 2015;3(2):e69.

36. Huh, J., et al., Effect of a mobile just-in-time implementation intention intervention on momentary smoking lapses in smoking cessation attempts among Asian American young adults. Translational Behavioral Medicine, 2020.

37. Bevona, C. and A.J. Sober, Melanoma incidence trends. Dermatologic clinics, 2002. 20(4): p. 589-95, vii.

38. Curado M-P, et al. Cancer incidence in five continents, vol. IX. International Agency for Research on Cancer: IARC Press; 2007.

39. Rogers HW, et al. Incidence estimate of nonmelanoma skin cancer (keratinocyte carcinomas) in the US population, 2012. JAMA Dermatol. 2015;151(10):1081-6.

40. Sutton A, Crew A, Wysong A. Redefinition of skin cancer as a chronic disease. JAMA Dermatol. 2016;152(3):255-6.

41. Kumar S, et al. Mobile health technology evaluation: the mHealth evidence workshop. Am J Prev Med. 2013;45(2):228-36.

42. Nilsen W, et al. Advancing the science of mHealth. J Health Commun. 2012;17(sup1):5-10.

43. Chakrabarti R. Principles of $m-H e a l t h$ survey design. Journal of Mobile Technology in Medicine. 2012;1(3):1-5.

44. Rodrigues AM, et al. Systematic and iterative development of a smartphone app to promote sun-protection among holidaymakers: design of a prototype and results of usability and acceptability testing. JMIR Research Protocols. 2017;6(6):e112.

45. Buller DB, et al. User-centered development of a smart phone mobile application delivering personalized real-time advice on sun protection. Translational behavioral medicine. 2013;3(3):326-34.

46. Hardeman $\mathrm{W}$, et al. A systematic review of just-in-time adaptive interventions (JITAIs) to promote physical activity. Int J Behav Nutr Phys Act. 2019;16(1):31.

47. Creswell, J.W. and C.N. Poth, Qualitative inquiry and research design: Choosing among five approaches. 2016: Sage publications.

48. Fogg, B., Mobile persuasion: 20 perspectives on the future of behavior change. 2007: Mobile Persuasion.

49. Fogg BJ. Persuasive technology: using computers to change what we think and do. Ubiquity. 2002;2002(December):5.

50. Katz SJ, Byrne S. Construal level theory of mobile persuasion. Media Psychol. 2013;16(3):245-71.

51. Johnson K, et al. Sun protection practices for children: knowledge, attitudes, and parent behaviors. Arch Pediatr Adolesc Med. 2001;155(8):891-6.

52. Altieri L, et al. Prevalence of sun protection behaviors in Hispanic youth residing in a high ultraviolet light environment. Pediatr Dermatol. 2018;35(1):e52-4.

53. Miller K, et al. SunSmart: evaluation of a pilot school-based sun protection intervention in Hispanic early adolescents. Health Educ Res. 2015;30(3):371-9.

54. Miller KA, et al. Patterns of sun protective behaviors among Hispanic children in a skin cancer prevention intervention. Prev Med. 2015;81:303-8.
55. Yip, J., K.J. Lee, and J. Lee, Design partnerships for participatory librarianship: A conceptual model for understanding librarians co-designing with digital youth. J Assoc Inf Sci Tech. 2020.

56. Cohen D. and B. Qualitative research guidelines project: Crabtree; 2006.

57. Pina, L.R., et al., How Latino children in the US engage in collaborative online information problem solving with their families. Proceedings of the ACM on Human-Computer Interaction, 2018. 2(CSCW): p. 1-26.

58. Kumar, P., et al., 'No Telling Passcodes Out Because They're Private' Understanding Children's Mental Models of Privacy and Security Online. Proceedings of the ACM on Human-Computer Interaction, 2017. 1(CSCW): p. 1-21.

59. Merriam, S.B. and E.J. Tisdell, Qualitative research: A guide to design and implementation. 2015: John Wiley \& Sons.

60. Birks M, Chapman Y, Francis K. Memoing in qualitative research: probing data and processes. J Res Nurs. 2008;13(1):68-75.

61. Stern RS, Weinstein MC, Baker SG. Risk reduction for nonmelanoma skin cancer with childhood sunscreen use. Arch Dermatol. 1986;122(5):537-45.

62. Baezconde-Garbanati LA, et al. Tamale Lesson: a case study of a narrative health communication intervention. J Commun Healthc. 2014;7(2):82-92.

63. Chan A, et al. Evaluation of fotonovela to increase human papillomavirus vaccine knowledge, attitudes, and intentions in a lowincome Hispanic community. BMC Res Notes. 2015;8(1):615.

64. Lamb RLB, et al. Evaluation of entertainment education strategies to promote cervical cancer screening and knowledge in Colombian women. J Cancer Educ. 2018;33(5):1094-101.

65. Ramirez OM, McCollough CA. "La Lotería"-using a culturally relevant mathematics activity with pre-service teachers at a family math learning event. Teaching for Excellence and Equity in Mathematics. 2012;4(1):24-33.

66. Bardzell, S. Feminist HCI: taking stock and outlining an agenda for design. in Proceedings of the SIGCHI conference on human factors in computing systems. 2010.

67. Ogbonnaya-Ogburu, I.F., et al. Critical Race Theory for HCI. in Proceedings of the $2020 \mathrm{CHI}$ Conference on Human Factors in Computing Systems (CHI'20). https://doi.org/10.1145/3313831. 3376392. 2020.

68. Berry B, McMullen T. Visual communication to children in the supermarket context: health protective or exploitive? Agric Hum Values. 2008;25(3):333-48.

69. Wyatt, T.H. and E. Hauenstein, Enhancing children's health through digital story. CIN: Computers, Informatics, Nursing, 2008. 26(3): p. 142-148.

70. Wyatt TH, Hauenstein EJ. Pilot testing okay with asthma TM: an online asthma intervention for school-age children. J Sch Nurs. 2008;24(3):145-50.

71. Küpper, A., U. Bareth, and B. Freese. Geofencing and background tracking-the next features in LBSs. in Proceedings of the 41th Annual Conference of the Gesellschaft für Informatik eV. 2011.

72. Rodriguez Garzon, S. and B. Deva. Geofencing 2.0: taking location-based notifications to the next level. in Proceedings of the 2014 ACM International Joint Conference on Pervasive and Ubiquitous Computing. 2014.

73. McNally, B., et al. Gains from participatory design team membership as perceived by child alumni and their parents. in Proceedings of the $2017 \mathrm{CHI}$ conference on human factors in computing systems. 2017.

Publisher's Note Springer Nature remains neutral with regard to jurisdictional claims in published maps and institutional affiliations. 